\title{
Estudio exploratorio de evaluación de riesgo en la salud de madres lactantes por consumo de pescado contaminado del río Cauca, en el Valle del Cauca (Colombia)*
}

Exploratory Study on the Evaluation of Health Risk in Lactating Mothers due to Contaminated Fish Consumption from the Cauca River, Valle del Cauca (Colombia)

Andrés Mauricio Zapata Rivera ${ }^{\text {a }}$

Universidad del Valle, Colombia

andres.zapata@correounivalle.edu.co

ORCID: http://orcid.org/0000-0002-6861-6771

Fabián Méndez

Universidad del Valle, Colombia

ORCID: http://orcid.org/0000-0002-5201-8822

Martha Isabel Páez

Universidad del Valle, Colombia

ORCID: http://orcid.org/0000-0002-3403-8440

Nathalie Abrahams Chow

Universidad del Valle, Colombia

ORCID: http://orcid.org/0000-0002-7452-8866

Jenny Ordoñez

Universidad del Valle, Colombia

ORCID: http://orcid.org/0000-0001-6021-0411

Yuly Paola Artunduaga

Universidad del Valle, Colombia

ORCID: http://orcid.org/0000-0002-9177-839X
DOI: https://doi.org/10.11144/Javeriana.ayd22-43.eeer Redalyc: http://www.redalyc.org/articulo.oa? id $=151560179002$

Fecha de recepción: 20 Julio 2018 Fecha de publicación: 30 Diciembre 2018

\section{Resumen:}

Se cuantificó la concentración de cadmio $(\mathrm{Cd})$, mercurio $(\mathrm{Hg})$ y plomo $(\mathrm{Pb})$ en tejido muscular de los peces Prochilodus magdalenae (bocachico) y Chaetostoma spp. (corroncho). Se estimó el riesgo por consumo de estos pescados en la salud de madres lactantes residentes en tres localidades ubicadas en la ribera del río Cauca (una rural y una urbana en el municipio de Cali, y una rural en el municipio de Yumbo). Se cuantificó el riesgo cancerígeno y no cancerígeno usando modelación probabilística con Crystal Ball 11.0Ò, aplicando el modelo de simulación Monte Carlo para cien mil iteraciones. Se obtuvieron las siguientes concentraciones promedio de metales: $\mathrm{Cd}=46 \mu \mathrm{g} / \mathrm{kg}-1, \mathrm{Hg}=235 \mu \mathrm{g} / \mathrm{kg}-1 \mathrm{y} \mathrm{Pb}=35 \mu \mathrm{g} / \mathrm{kg}-1$. Para el mercurio, se encontró que en las dos localidades ubicadas en Cali, los valores de riesgo no cancerígeno y la ingesta semanal tolerable están por encima de los límites máximos permitidos por la Agencia de Protección Ambiental de los Estados Unidos.

Palabras clave: contaminación química, estudios poblacionales en salud pública, metales pesados, población, riesgo ambiental, río Cauca, salud ambiental.

\section{Abstract:}

Cadmium $(\mathrm{Cd})$, mercury $(\mathrm{Hg})$ and lead $(\mathrm{Pb})$ concentration was quantified in the fish Prochilodus magdalenae (bocachico) and Chaetostoma spp. (corroncho). The health risk due to consuming this fish was estimated in lactating mother living in three locations along the Cauca River. Carcinogenic and non-carcinogenic risk was quantified by using probabilistic modeling with Crystal Ball 11.0Oे and applying the Monte Carlo simulation model for 100,000 iterations. The average concentration was measured for the

Notas de autor:

$$
\text { a Autor de correspondencia. Correo electrónico: andres.zapata@correounivalle.edu.co }
$$


following metals: $\mathrm{Cd}=46 \mu \mathrm{g} / \mathrm{kg}-1, \mathrm{Hg}=235 \mu \mathrm{g} / \mathrm{kg}-1$, and $\mathrm{Pb}=35 \mu \mathrm{g} / \mathrm{kg}-1$. Regarding the mercury, in two locations in Cali, it was found that the values for non-carcinogenic risk and tolerable weekly consumption are above maximum limits allowed by the USEPA.

Keywords: chemical contamination, heavy metals, population, public-health population studies, environmental risk, environmental health, Cauca River.

\section{Introducción}

En las últimas décadas, se ha encontrado un incremento de los agentes físicos, químicos y microbiológicos, provenientes de las actividades humanas que contaminan las fuentes de agua. Esto ha convertido a especies acuáticas, como los peces, en vías de exposición a diversos contaminantes, como los metales pesados. Diferentes estudios (El-Moselhy, Othman, El-Azem y El-Metwally, 2014; Kwok et al., 2014; Monroy, Maceda y De Sostoa, 2014) han reportado la presencia de metales como cobre, zinc, plomo, cadmio, hierro, manganeso y mercurio, en agua, sedimentos y especies de peces en distintos ecosistemas acuáticos alrededor del mundo. Algunos de estos metales pesados tienen el agravante de ser asociados con ciertos tipos de cáncer (Pollack et al., 2014), enfermedades del sistema nervioso central, endocrino y reproductivo (Burbure et al., 2006), malformaciones congénitas (Office of Research and Development, National Center for Environmental Assessment, 2001), enfermedades renales (Office of Research and Development, National Center for Environmental Assessment, 1987), detrimento del desarrollo mental de los niños, disminución en el cociente intelectual y problemas de comportamiento (Office of Research and Development, National Center for Environmental Assessment, 2014). Esto los constituye en una de las principales causas de deterioro de los ecosistemas acuáticos e incrementa los riesgos potenciales de detrimento de la salud de los animales y los humanos que se relacionan con ellos, principalmente mujeres y niños en etapa de gestación y lactancia (Al-Saleh, Shinwari, Mashhour, Mohamed y Rabah, 2011). En Colombia, los dos ríos más importantes, el Magdalena y el Cauca, no son ajenos a esta problemática. Los estudios realizados en estos cuerpos de agua han evidenciado la presencia de metales pesados, como arsénico, mercurio, cromo, cadmio, plomo y zinc, entre otros, en peces de estos ríos (Alvarez, Jessick, Palacio y Kolok, 2012; Mancera y Álvarez, 2006). El río Cauca y sus afluentes se caracterizan por ser receptores de las descargas de aguas residuales domésticas de las poblaciones ubicadas a lo largo de su recorrido. Esto incluye a los mayores centros urbanos del suroccidente colombiano (Popayán, Cali y Yumbo). Adicionalmente, debido al crecimiento de la minería ilegal y de la industria, es receptor de desechos y aguas industriales, en los que se han identificado metales pesados y pesticidas, que han tenido graves efectos sobre la biota acuática. Vivas et al. (2014) evaluaron la mutagenicidad del agua del río Cauca asociada a metales pesados, en su paso por la zona urbana de la ciudad de Santiago de Cali. Los resultados mostraron índices de mutagenicidad (IM) positivos (IM > 2,0) en el sector del corregimiento El Hormiguero (IM = 3,6), en la desembocadura del Canal Colector Sur $(\mathrm{IM}=2,9)$ y en la desembocadura del río Cali $(\mathrm{IM}=2,7)$, los dos últimos son afluentes del río Cauca. Esta problemática ha despertado el interés en la evaluación del riesgo para la salud de las poblaciones que se relacionan directamente con los ríos contaminados, especialmente, aquellas cuya dieta depende directamente del consumo de pescado extraído de estas fuentes hídricas (Echeverry, Zapata, Páez, Méndez y Peña, 2015; Raissy y Ansari, 2014; Storelli, 2008). La evaluación del riesgo cuantifica el impacto de los contaminantes en la salud de las personas, por lo que es una herramienta valiosa para la discusión sobre la naturaleza y el alcance del riesgo y el potencial deterioro de la salud de la población, fundamentalmente para implementar las acciones correctivas necesarias para mitigar el riesgo en las poblaciones evaluadas (Páez et al., 2011). En consecuencia, se desarrolló un estudio exploratorio para evaluar el riesgo en la salud por consumo de pescado del río Cauca, contaminado con cadmio $(\mathrm{Cd})$, mercurio $(\mathrm{Hg})$ y plomo $(\mathrm{Pb})$, en 22 mujeres lactantes entre los 15 y 29 años de edad. Para la evaluación, se siguió la metodología de simulación probabilística Monte Carlo para cien mil iteraciones y el modelo de evaluación de riesgo en salud de la Agencia de Protección Ambiental de los Estados Unidos (Office 
of Research and Development, National Center for Environmental Assessment, 1989). La zona de estudio estuvo comprendida por tres localidades ubicadas sobre el margen del río Cauca (una rural y una urbana en el municipio de Cali y una rural en el municipio de Yumbo), todas ubicadas en el departamento del Valle del Cauca (Colombia).

\section{Materiales y métodos}

\section{Ubicación y caracterización de la zona de estudio}

El muestreo de peces se realizó en el río Cauca, específicamente en el corregimiento de El Hormiguero, al suroriente de la ciudad de Cali (rural); en el jarillón de la Comuna 6, en el oriente de esta ciudad (urbana), y en la vereda El Paso de la Torre, en el municipio de Yumbo (rural). Estas localidades se caracterizan por poseer población con ingresos económicos bajos (menos de 100 dólares mensuales), un nivel educativo entre primaria y secundaria, consumo frecuente de pescado del río Cauca y por colindar, al sur de Cali, con una zona de expansión, en donde se encuentra ubicado el antiguo vertedero de residuos sólidos municipales, conocido como Navarro. Este vertedero, operó por cerca de 40 años, hasta el año 2008, y en él se depositaban, en los últimos años de funcionamiento, cerca de $1700 \mathrm{t} /$ día $^{-1}$ de residuos. En este vertedero y en el suelo aledaño se reportó la presencia de metales pesados en el año 2007 (Mendoza, González y Benítez, 2007).

En la zona de estudio se encontraron 22 madres lactantes ( 5 en El Hormiguero, 13 en la Comuna 6 y 4 en la vereda El Paso de la Torre), las cuales correspondían al total de madres lactantes residentes en la zona en el momento del estudio. Las madres fueron identificadas gracias a que en el jarillón de Floralia y en la vereda El Paso de la Torre el número total de habitantes no sobrepasa las doscientas personas, por lo que se hizo un acercamiento con los líderes de cada comunidad, quienes ayudaron a su localización. En el corregimiento El Hormiguero, que es una población con aproximadamente cinco mil habitantes, la localización se hizo a través de la institución de salud del lugar, gracias a que contaban con un registro detallado de las madres y su lugar de residencia. Las madres lactantes asistían regularmente a los controles médicos en el centro de salud colaborador, lo que garantizó su presencia durante los procesos necesarios para el estudio, tales como la toma del consentimiento informado y la información acerca de la toma de muestras biológicas. Además de la lactancia, se usaron como criterios de inclusión en el estudio que la madre tuviera su vivienda a no mas de 20 $\mathrm{m}$ de la ribera del río y que consumiera con regularidad pescado del río Cauca. El estudio contó con el aval del comité de ética de la Universidad del Valle. En la figura 1 se muestra el área de estudio. 

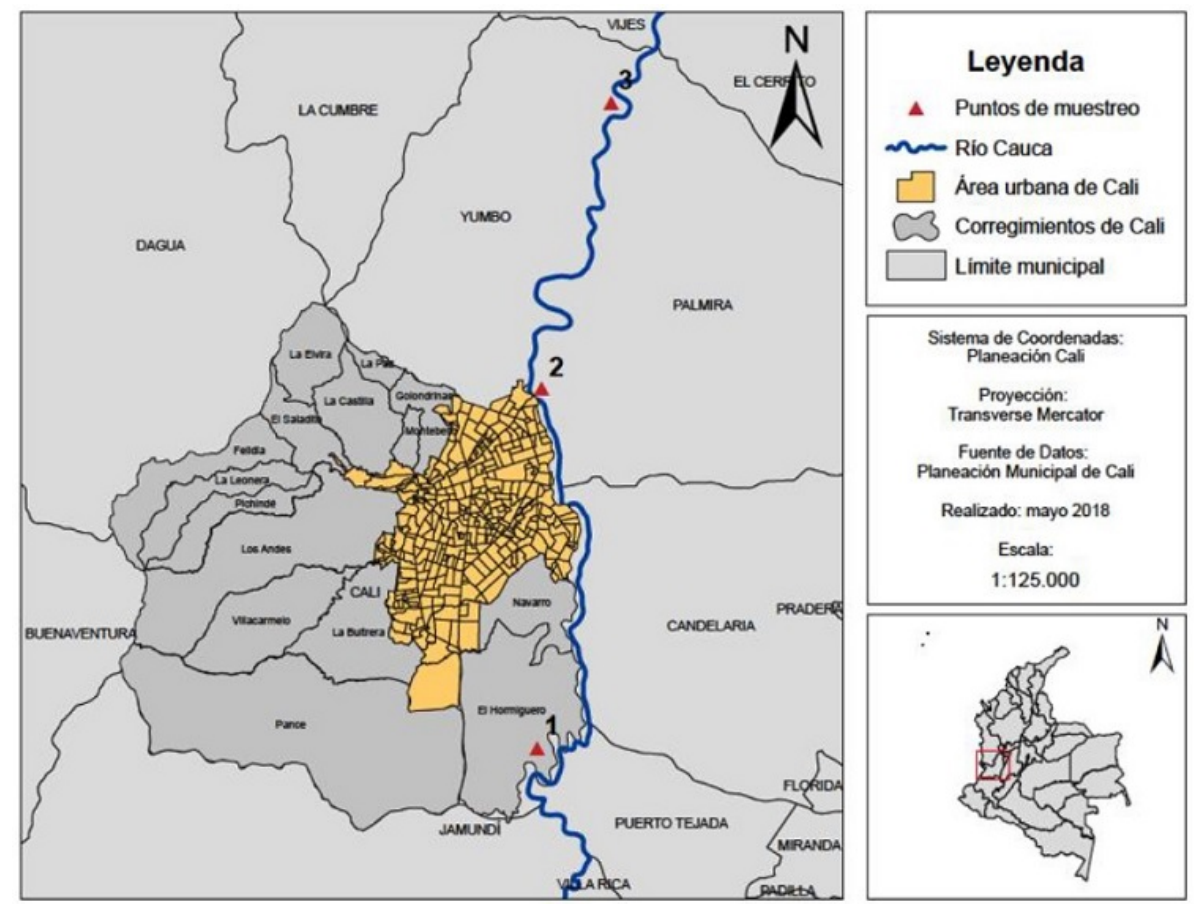

FIGURA 1

Zona de estudio: corregimiento de El Hormiguero (1), jarillón de la Comuna 6 (2) y vereda El Paso de la Torre (3) Fuente: elaborada por Andrés Mauricio Zapata Rivera, 2018

\section{Toma de muestras de pescado}

Se realizó un muestreo puntual durante una temporada atípica de alta lluvia entre los meses de diciembre de 2014 y febrero de 2015. Se colectaron 7 muestras de pescado en cada punto, para un total (n) de 21, de forma aleatoria, y con la ayuda de pescadores de la zona para la captura, transporte y almacenamiento de las muestras, se aplicó la guía para el muestreo, preparación y análisis de contaminantes en muestras ambientales del programa de monitoreo costero Repcar (Programa Ambiental del Caribe, 2008). De las 21 muestras, 11 correspondieron a Prochilodus magdalenae (bocachico) (B) y 10 a Chaetostoma spp. (corroncho) (C), según la abundancia en cada sitio. El número de muestras se calculó para estimar la media de las concentraciones siguiendo la metodología de la distribución t de student para un nivel de confianza del 95\%, una desviación estándar del 2,84 y un nivel de error aceptable del 3\% (Mejías y Jerez, 2006).

\section{Preservación y tratamiento de las muestras}

La matriz de estudio fue el músculo del pescado, debido a que es la parte preferida de consumo de la población y una de las partes de bioacumulación de los metales. Las muestras se liofilizaron para su preservación a -40 ${ }^{\circ} \mathrm{C}$ durante 32 horas. La liofilización se llevó a cabo en el laboratorio de la Escuela de Ingeniería de Alimentos de la Universidad del Valle, utilizando un liofilizador marca Labconco, fabricado por Labconco Corporation, en Kansas City (Estados Unidos). A las muestras liofilizadas se les realizó la extracción de plomo y cadmio a través de calcinación, siguiendo los lineamientos sugeridos por Gil, Torres, Harvey y Esteves (2006). Para el mercurio se utilizó la digestión ácida asistida por microondas, según las especificaciones del método Usepa 3052 (Office of Research and Development, National Center for Environmental Assessment, 1996). 


\section{Cuantificación de metales pesados}

La cuantificación de metales pesados se realizó siguiendo los lineamientos del método Usepa 7010 (Office of Research and Development, National Center for Environmental Assessment, 1998), utilizando espectrometría de absorción atómica. El cadmio y el plomo se analizaron por absorción atómica por horno de grafito, mientras que el mercurio se cuantificó por vapor frío. Se utilizó un equipo Shimadzu AA 6300, fabricado por Shimadzu Inc., en Riverwood Drive (Columbia, Estados Unidos). Los reactivos utilizados fueron grado analítico y los estándares para $\mathrm{Cd}, \mathrm{Hg}$ y $\mathrm{Pb}$ fueron marca Sigma Aldrich (de St. Louis, Estados Unidos) con una concentración cada uno de $1000 \mathrm{mg} / \mathrm{L}^{-1}$ en ácido nítrico. Se obtuvieron los siguientes límites de detección: $\mathrm{Cd}=0,16 \mu \mathrm{g} / \mathrm{kg}^{-1}, \mathrm{Hg}=0,81 \mu \mathrm{g} / \mathrm{kg}^{-1}$ y $\mathrm{Pb}=1,18 \mu \mathrm{g} / \mathrm{kg}^{-1}$. La desviación estándar fue: para $\mathrm{Pb}$ $=1,30$, para $\mathrm{Cd}=0,53$ y para $\mathrm{Hg}=2,67$; mientras que los coeficientes de variación para estos metales fueron: $2,9 \%, 0,5 \%$ y $2,4 \%$, respectivamente.

\section{Escenario de la evaluación de riesgo para la salud humana}

En este estudio, los pescados extraídos del río Cauca se consideran como la vía a través de la cual los contaminantes llegan a la población, mientras que su ingestión es la ruta por la que los contaminantes llegan al sistema gastrointestinal. La población receptora objeto de este estudio de evaluación de riesgo fue una muestra propositiva de 22 madres lactantes, entre los 15 y 29 años de edad, expuestas a los metales pesados por el consumo de pescado del río.

Para la evaluación de riesgo ambiental para la salud humana se utilizaron los modelos de la Agencia de Protección Ambiental de los Estados Unidos. Según estos, el riesgo es una función de la toxicidad de la sustancia peligrosa evaluada, la magnitud y el tiempo de exposición, siendo esta última una medida de la calidad y la cantidad del contacto entre la sustancia y el organismo expuesto (Office of Research and Development, National Center for Environmental Assessment, 1989).

\section{Ruta de exposición por ingestión}

La ingesta (I) se determinó utilizando la ecuación genérica usada para el cálculo de la exposición (ecuación 1), expresada a menudo como un consumo diario promedio con base en el peso corporal (unidades de miligramos por kilogramo de peso corporal por día $\left.[\mathrm{mg}(\mathrm{kg} / \mathrm{día})]^{-1}\right)$. La ecuación genérica usada fue la siguiente (Office of Research and Development, National Center for Environmental Assessment, 1989):

$$
I=\frac{C \times I R \times C F \times E F \times E D}{B W \times A T}
$$

Donde:

$\mathrm{C}=$ concentración en la muestra $(\mathrm{mg} / \mathrm{kg}-1)$

$\mathrm{IR}=$ tasa de ingestión de la muestra ( $\mathrm{mg} /$ día-1)

$\mathrm{CF}=$ factor de conversión $(1 \mathrm{E}-06 \mathrm{~kg} / \mathrm{mg}-1)$

$\mathrm{EF}=$ frecuencia de exposición (días/año-1)

$\mathrm{ED}=$ tiempo exposición (años)

$\mathrm{BW}=$ peso corporal $(\mathrm{kg})$

$\mathrm{AT}=$ tiempo promedio (días) 


\section{Obtención de los parámetros del modelo}

Se consideró como población expuesta al grupo de 22 mujeres lactantes residentes en la zona de estudio. La dieta de estas mujeres incluye el consumo frecuente de pescado extraído del río Cauca. A estas mujeres se les aplicó una encuesta de hábitos relacionados con las rutas y vías de exposición de interés. Dicha encuesta se ha utilizado en otros estudios y se adaptó culturalmente para la población de la zona de estudio. La encuesta mostró que el consumo de pescado promedio reportado fue de $14.000 \mathrm{mg} / \mathrm{día}^{-1}$, con valores máximos de $26.300 \mathrm{mg} / \mathrm{día}^{-1}$ y mínimos de $2360 \mathrm{mg} / \mathrm{día}^{-1}$. La frecuencia de exposición fue de 98 días/año ${ }^{-1}$, con un máximo de 162 días/año ${ }^{-1}$ y un mínimo de 41 días/año ${ }^{-1}$; la duración promedio de la exposición fue de 21 años, con un máximo de 29 años y un mínimo de 15 años, valores que correspondieron con la edad de las madres participantes en el estudio. Los valores máximo y mínimo del peso corporal de las mujeres de la zona fueron de $62,8 \mathrm{~kg}$ y $50,5 \mathrm{~kg}$, con un promedio de $56,8 \mathrm{~kg}$. Todas estas unidades son requeridas en el modelo Usepa.

\section{Caracterización y cálculo del riesgo}

El cálculo del riesgo por sustancia de efectos tóxicos no carcinógenos (REN) se realizó por el cociente (REN= $\mathrm{I} / \mathrm{R} f \mathrm{D})$ del valor de la ingesta calculada (I) entre una dosis referencial específica $(\mathrm{R} f \mathrm{D})$ para esa ruta. Si el $\mathrm{REN} \geq 1$, se considera que existe un riesgo para la salud inaceptable y habría que tomar medidas correctivas (Office of Research and Development, National Center for Environmental Assessment, 1989). Para el riesgo por efectos tóxicos carcinogénicos, se utilizó el factor de pendiente de cáncer (CSF, por sus siglas en inglés) y se determinó el producto de la ingesta por el factor de cáncer de cada contaminante evaluado ( $\mathrm{RC}=\mathrm{Ix} \mathrm{CSF}$ ). El riesgo cancerígeno puede tomar un valor del orden de $1 \times 10^{-4}, 1 \times 10^{-5}$ o $1 \times 10^{-6}$. Estos valores dependen de cuán conservador se quiera ser en la evaluación del riesgo, y suelen estar ligados al riesgo mismo de ocurrencia de un impacto (cantidad de vías de exposición, tipo de zonificación, etc.). Un nivel de riesgo de $1 \times 10^{-\mathrm{N}}$ indica que hasta un caso de cáncer entre cada $10^{\mathrm{N}}$ habitantes es producto de causas ajenas al escenario evaluado, los casos adicionales sobre este umbral serán atribuidos al escenario bajo estudio.

\section{Evaluación de riesgo en salud: método probabilístico}

Según la Office of Research and Development, National Center for Environmental Assessment, el cálculo probabilístico de riesgo implica un muestreo aleatorio, a partir del cual se genera cada una de las distribuciones de las variables incluidas en la exposición, siendo el análisis Monte Carlo uno de los métodos probabilísticos más ampliamente utilizados. La aplicación del análisis probabilístico en evaluaciones de riesgo para la salud humana es un desarrollo relativamente nuevo que se ha facilitado por el desarrollo de técnicas de muestreo estadístico y el aumento en la velocidad y la capacidad de equipos modernos que pueden soportar los requerimientos computacionales de una simulación Monte Carlo (Echeverry et al., 2015). Por tal razón, para el cálculo del riesgo se siguieron los lineamientos de un modelo probabilístico usando el software Crystal Ball 11.0 (desarrollado por Decisioneering Inc., ubicada en Denver [Colorado, Estados Unidos]), con simulaciones tipo Monte Carlo, para cien mil iteraciones, basados en los tipos de distribución de probabilidad de cada variable. Para ello, se aplicó la prueba de normalidad Shapiro Wilk ( $<50$ y p $>0,05)$ empleando el software Statistica v. 8.0 (de StatSoft, de Polonia). Posteriormente, se aplicó la prueba chi cuadrado, para determinar la distribución a la que se ajustaban los datos. La prueba de Shapiro Wilk mostró que no hay normalidad en ninguna de las variables de la ecuación de riesgo. Mientras que la prueba chi estableció que todas las variables se ajustan a una distribución rectangular. Por lo tanto, se trabajó con los valores máximos 
y mínimos de cada una de las variables del modelo Usepa. Los valores de la dosis de referencia $(\mathrm{R} f \mathrm{D})$ para cadmio, mercurio y plomo fueron $5,00 \times 10^{-4}, 1,00 \times 10^{-4}$ y $3,57 \times 10^{-3}\left(\mathrm{mg} / \mathrm{kg}^{-1} / \mathrm{dia}^{-1}\right)$, respectivamente, mientras que el factor de cáncer (CSF) para mercurio fue de $3,50 \times 10^{-2}\left(\mathrm{mg} / \mathrm{kg}^{-1} / \mathrm{dia}^{-1}\right)$.

\section{Ingesta semanal tolerable (PTWI)}

Como complemento a la evaluación probabilística del riesgo, se realizó una comparación directa entre la ingesta de contaminantes a través del pescado y la ingesta semanal tolerable provisional (PTWI, por sus siglas en inglés), establecida por el Comité Científico de Expertos en Aditivos Alimentarios (European Food Safety Authority, 2004). La PTWI se expresa como "la dosis de un producto que puede ser ingerida cada semana por un individuo durante toda su vida sin riesgo para la salud”. Esta cantidad se expresa en mg.kg ${ }^{-1}$ de peso corporal. El principal mensaje de la PTWI es que no existe un riesgo importante cuando el producto químico se ingiere en niveles similares o inferiores a esa PTWI.

\section{Resultados}

\section{Concentración de metales pesados en peces}

Para los resultados del contenido de plomo, cadmio y mercurio en las muestras de pescado, se consignó el promedio de las lecturas de cada muestra, las cuales fueron analizadas por triplicado. Las especies de peces Prochilodus magdalenae (bocachico) y Chaetostoma spp. (corroncho) procedentes del río Cauca presentan bioacumulación de estos tres metales pesados. Este resultado es consistente con los obtenidos en estudios previos realizados en este mismo río en los años 2006 y 2014 (Mancera y Álvarez, 2006; Vivas et al., 2014).

$\mathrm{Al}$ comparar los resultados experimentales con los valores máximos permitidos para estos metales en las normas europea y de la Organización de las Naciones Unidas para la Agricultura y la Alimentación (FAO, 2013) y Mercosur (2014), se pudo establecer que en todas las muestras el contenido de plomo estuvo por debajo de los límites máximos permitidos, $\left(<300 \mathrm{mg} / \mathrm{kg}^{-1}\right)$. En contraste, en cinco de las muestras recolectadas en la vereda El Paso de la Torre y en una de las muestras de la Comuna 6 se hallaron valores de cadmio por encima del límite máximo permitido en la normatividad. Específicamente, las normas establecen un contenido máximo de cadmio de $50 \mathrm{mg} / \mathrm{kg}^{-1}$, y las muestras analizadas presentaron los siguientes valores: la muestra 8B de la Comuna 6, 137,60 mg/ $\mathrm{kg}^{-1}$; las muestras 15C, 16C, 19C, 20C y 21C de la vereda El Paso de la Torre presentaron los valores de $131,30 \mathrm{mg} / \mathrm{kg}^{-1}, 55,90 \mathrm{mg} / \mathrm{kg}^{-1}, 86,58 \mathrm{mg} / \mathrm{kg}^{-1}, 67,08 \mathrm{mg} / \mathrm{kg}^{-1}$ y $64,86 \mathrm{mg} / \mathrm{kg}^{-1}$, respectivamente. Por lo anterior, se puede colegir que estas muestras no cumplen con los requerimientos de las normas y que el pescado en estas dos ubicaciones no es apto para su consumo. Para el mercurio, se encontró que las muestras de pescado tomadas en las tres ubicaciones cumplen con las normas. Sin embargo, las muestras $4 \mathrm{~B}$ y $6 \mathrm{~B}$, tomadas en el corregimiento de El Hormiguero, y las muestras 8B, 9B y $10 \mathrm{~B}$, tomadas en la Comuna 6, presentan valores de contenido de mercurio cercanos al límite máximo permitido. Los resultados experimentales fueron los siguientes: $411,42 \mathrm{mg} / \mathrm{kg}^{-1}, 436,60 \mathrm{mg} / \mathrm{kg}^{-1}, 422,90 \mathrm{mg} /$ $\mathrm{kg}^{-1}, 422,10 \mathrm{mg} / \mathrm{kg}^{-1}$ y $490,42 \mathrm{mg} / \mathrm{kg}^{-1}$, respectivamente, y las normas establecen un límite máximo de $<500$ $\mathrm{mg} / \mathrm{kg}^{-1}$. 


\section{Resultados de la evaluación de riesgo para la salud}

Los resultados probabilísticos obtenidos para la evaluación del riesgo por efectos no carcinogénicos y carcinogénicos debido a la ingesta de pescado del río Cauca con contenido de cadmio, mercurio y plomo se registran en la tabla 1.

TABLA 1

Valores mínimo y máximo del riesgo no carcinogénico y carcinogénico en cada ubicación

\begin{tabular}{|c|c|c|c|c|c|c|}
\hline \multirow[t]{2}{*}{ Lugar de muestreo } & \multicolumn{2}{|c|}{$\begin{array}{c}\text { Plomo } \\
\text { (no cancerígeno) }\end{array}$} & \multicolumn{2}{|c|}{$\begin{array}{c}\text { Cadmio } \\
\text { (no cancerígeno) }\end{array}$} & \multicolumn{2}{|c|}{$\begin{array}{c}\text { Mercurio } \\
\text { (no cancerígeno) }\end{array}$} \\
\hline & Mínimo & Máximo & Mínimo & Máximo & Mínimo & Máximo \\
\hline El Hormiguero & $-6,26 \times 10^{-3}$ & $1,10 \times 10^{-2}$ & $-4,42 \times 10^{-3}$ & $1,24 \times 10^{-2}$ & $-2,38 \times 10^{-2}$ & $1,59^{*}$ \\
\hline Comuna 6 & $-4,22 \times 10^{-3}$ & $8,21 \times 10^{-3}$ & $-2,36 \times 10^{-2}$ & $9,12 \times 10^{-3}$ & $1,05^{*}$ & $3,13^{*}$ \\
\hline El Paso de La Torre & $-3,88 \times 10^{-3}$ & $8,45 \times 10^{-3}$ & $-2,00 \times 10^{-2}$ & $5,09 \times 10^{-2}$ & $1,85 \times 10^{-3}$ & $3,25 \times 10^{-1}$ \\
\hline \multicolumn{7}{|l|}{ Cancerígeno } \\
\hline El Hormiguero & N.A. & N.A. & N.A. & N.A. & $-2,44 \times 10^{-6}$ & $6,85 \times 10^{-6}$ \\
\hline Comuna 6 & N.A. & N.A. & N.A. & N.A. & $-4,98 \times 10^{-6}$ & $1,31 \times 10^{-5}$ \\
\hline El Paso de la Torre & N.A. & N.A. & N.A. & N.A. & $-6,22 \times 10^{7}$ & $1,64 \times 10^{-6}$ \\
\hline
\end{tabular}

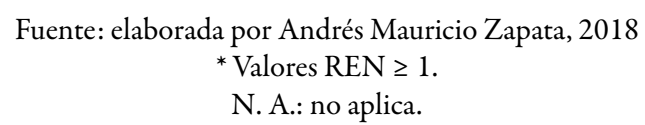

La sumatoria del riesgo para cada punto y contaminante fue la siguiente: para el corregimiento de El Hormiguero, la Comuna 6 y la vereda El Paso de La Torre, la sumatoria del valor máximo del riesgo no cancerígeno fue de $1,61 \mathrm{E}+00^{*}, 3,15 \mathrm{E}+00^{*}$ y $3,8 \mathrm{E}-01$, respectivamente. Este corresponde al peor escenario posible que se puede presentar en la zona de estudio.

Para el riesgo cancerígeno se obtuvieron las siguientes sumatorias: para el corregimiento de El Hormiguero, 6,85E - 6: para la Comuna 6, 1,31E - 5, y para la vereda El Paso de La Torre, 1,64E - 06.

Estos resultados indican que los valores máximos del riesgo no cancerígeno por el consumo de pescado en el corregimiento de El Hormiguero y la Comuna 6, en la ciudad de Cali, están por encima de los valores máximos permitidos (REN $\geq 1)$ por la Agencia de Protección Ambiental de los Estados Unidos. Esto significa que existe un riesgo inaceptable para la salud de la población, por lo que se deben tomar las medidas necesarias para mitigar este riesgo. El riesgo carcinogénico se encuentra en los rangos permitidos de $<10^{-5}$, por lo que se considera tolerable. Los resultados son acordes con estudios que demuestran que las comunidades asentadas en zonas costeras o en riberas basan su dieta en un mayor consumo de pescado, por lo que pueden consumir entre un 40\% y un 50\% más pescado que el resto de la población (Castro y Méndez, 2008), lo que finalmente incrementa el riesgo para la salud de las poblaciones expuestas (Vieira, Morais, Ramos, Delerue y Oliveira, 2011; Alvarez, Kolok, Jimenez, Granados y Palacio, 2012; Garcia et al., 2011).

\section{Comparación de la ingesta de contaminantes a través del pescado con la ingesta semanal tolerable provisional (PTWI)}

En la tabla 2 se presentan los resultados de la ingesta semanal tolerable de los metales evaluados. 
TABLA 2

Resultados de la ingesta semanal tolerable (PTWI) para cadmio, mercurio y plomo

\begin{tabular}{lrrrr}
\hline \multirow{2}{*}{ Lugar } & $\begin{array}{c}\text { Identificación } \\
\text { de la muestra }\end{array}$ & $\begin{array}{c}\text { Plomo } \\
\left(\mu \mathrm{g} / \mathrm{kg}^{-1}\right)\end{array}$ & $\begin{array}{r}\text { Cadmio } \\
\left(\mu \mathrm{g} / \mathrm{kg}^{-1}\right)\end{array}$ & $\begin{array}{r}\text { Mercurio } \\
\left(\mu \mathrm{g} / \mathrm{kg}^{-1}\right)\end{array}$ \\
\hline El & $1 \mathrm{~B}$ & 1,411 & 0,359 & 3,995 \\
\cline { 2 - 5 } Hormiguero & 2B & 0,435 & 0,298 & $5,822^{*}$ \\
\cline { 2 - 5 } & 3B & 0,000 & 0,273 & $5,700^{*}$ \\
\cline { 2 - 5 } & 4B & 0,000 & 0,367 & $6,618^{*}$ \\
\cline { 2 - 5 } & 5B & 0,098 & 0,307 & $6,300^{*}$ \\
\cline { 2 - 5 } & 6B & 0,000 & 0,328 & $7,024^{*}$ \\
\hline Comuna 6 & $7 \mathrm{~B}$ & 0,292 & 0,441 & 3,695 \\
\cline { 2 - 5 } & 8B & 0,489 & 2,214 & $6,803^{*}$ \\
\cline { 2 - 5 } & 9B & 0,081 & 0,785 & $6,791^{*}$ \\
\cline { 2 - 5 } & $10 \mathrm{~B}$ & 0,000 & 0,789 & $7,889^{*}$ \\
\cline { 2 - 5 } & $11 \mathrm{~B}$ & 0,000 & 0,384 & 3,642 \\
\cline { 2 - 5 } & $12 \mathrm{C}$ & 0,221 & 0,513 & 1,758 \\
\cline { 2 - 5 } & $13 \mathrm{C}$ & 0,856 & 0,505 & 1,394 \\
\hline El Paso de La & $14 \mathrm{C}$ & 0,327 & 0,327 & 1,456 \\
\cline { 2 - 5 } Torre & $15 \mathrm{C}$ & 0,294 & 2,112 & 1,331 \\
\cline { 2 - 5 } & $16 \mathrm{C}$ & 0,046 & 0,899 & 1,454 \\
\cline { 2 - 5 } & $17 \mathrm{C}$ & 1,137 & 0,524 & 1,335 \\
\cline { 2 - 5 } & $18 \mathrm{C}$ & 0,977 & 0,415 & 1,572 \\
\cline { 2 - 5 } & $19 \mathrm{C}$ & 0,098 & 1,393 & 1,935 \\
\cline { 2 - 5 } & 20C & 0,800 & 1,079 & 1,574 \\
\cline { 2 - 5 } & $21 \mathrm{C}$ & 1,407 & 1,043 & 1,273 \\
\hline & & & & \\
\hline & & &
\end{tabular}

Fuente: elaborada por Andrés Mauricio Zapata, 2018

* Valor por encima de lo estipulado para la ingesta semanal tolerable provisional.

Se comparó la ingesta directa de contaminantes a través del pescado con la ingesta semanal tolerable provisional (PTWI), establecida por el Comité Mixto FAO/OMS de Expertos en Aditivos Alimentarios. Los valores de la PTWI para cadmio, plomo y mercurio son $7 \mu \mathrm{g} / \mathrm{kg}^{-1}, 25 \mu \mathrm{g} / \mathrm{kg}^{-1}$ y $5 \mu \mathrm{g} / \mathrm{kg}^{-1} \mathrm{de}$ peso corporal, respectivamente.

En las zonas evaluadas, se encontró, según la encuesta poblacional, que las mujeres poseen un peso corporal promedio de $62,16 \mathrm{~kg}$ y consumen pescado más de una vez a la semana (en promedio $1,40 \mathrm{E} 04 \mathrm{mg} / \mathrm{día}^{-1}$ ). Este 
consumo de pescado, el cual es parte fundamental de la dieta de las madres lactantes, ocasiona que los valores de la PTWI para mercurio en las muestras 2B, 3B, 4B, 5B y 6 B, del corregimiento de El Hormiguero, y las muestras 8B, 9B y 10B, de la Comuna 6, en el municipio de Cali, sobrepasen el límite máximo permisible de ingesta semanal. Estos resultados, sumados a los obtenidos en la evaluación del riesgo para la salud, permiten concluir que las mujeres residentes en la zona de estudio tienen una alta probabilidad de desarrollar los efectos crónicos tóxicos asociados al mercurio (Márquez et al., 2016), entre los que se incluyen enfermedades del sistema nervioso central, cáncer y malformaciones congénitas de la progenie (Duque, Marrugo, Suárez y Olivares, 2016).

\section{Discusión}

Los resultados obtenidos dejan en evidencian la grave problemática ambiental asociada al deterioro de la cuenca del río Cauca, lo que demanda el desarrollo de nuevas investigaciones en las que se evalúe el riesgo asociado a rutas de exposición como el contacto dérmico. Esta última ruta de exposición cobra importancia debido a que la zona de estudio está conformada por sectores deprimidos de los municipios de Cali y Yumbo, los cuales no cuentan con servicios de agua potable ni alcantarillado, lo que obliga a los habitantes de la zona a recurrir al río para satisfacer sus necesidades cotidianas, como el aseo del hogar, el aseo personal y la preparación de alimentos.

Los resultados de este estudio también muestran que los pescados de las especies Prochilodus magdalenae y Chaetostoma spp. presentan bioacumulación de cadmio, mercurio y plomo en su tejido muscular, lo que concuerda con resultados de estudios similares desarrollados en Colombia en el río Magdalena, (Alvarez, Kolok et al., 2012); la región de La Mojana, al norte del país (Marrugo, Verbel, Ceballos y Benitez, 2008), y los ríos Nechí y La Miel (Alvarez, Kolok et al., 2012). Diferentes autores coinciden en que, en Colombia, la minería artesanal e ilegal de extracción de oro es una de las principales causas de contaminación de las fuentes hídricas y de las especies acuáticas presentes en ellas (Cordy et al., 2011; Suárez y Aristizabal, 2013; Marrugo, Benitez y Olivero, 2008). Al respecto, las personerías del municipio de Cali y del departamento del Cauca coinciden en que el Cauca y el Valle del Cauca son departamentos que han evidenciado un gran incremento de la minería ilegal, particularmente en la zona del Parque Nacional Natural Los Farallones de Cali y en el norte del departamento del Cauca, con la consecuente contaminación con mercurio de las fuentes hídricas, como los ríos Cali y Meléndez, en la ciudad de Cali, y los ríos Ovejas, Teta y Quinamayó, en el norte del departamento del Cauca, todos tributarios del río Cauca. Por lo tanto, los resultados obtenidos en este estudio constituyen una evidencia del impacto de la minería ilegal en la zona y de su posterior impacto en la salud de las poblaciones ribereñas.

Las concentraciones de cadmio se encuentran por encima de los límites máximos permitidos $\left(50 \mathrm{mg} / \mathrm{kg}^{-1}\right)$ por las normas europea, FAO y Mercosur, mientras que las de mercurio están ligeramente por debajo de los valores establecidos en estas normas $\left(<500 \mathrm{mg} / \mathrm{kg}^{-1}\right)$. Los resultados de la evaluación de riesgo cancerígeno y no cancerígeno muestran que en el corregimiento de El Hormiguero y en la Comuna 6 el riesgo no cancerígeno presenta valores intolerables, por lo que se deben implementar medidas urgentes de mitigación del riesgo. Asimismo, los valores de la PTWI muestran que el consumo semanal de mercurio asociado a la ingesta de pescado supera los valores establecidos $\left(<5 \mu \mathrm{g} / \mathrm{kg}^{-1}\right.$ de peso corporal) por el Comité Mixto FAO/OMS de Expertos en Aditivos Alimentarios. A pesar de las desventajas por el consumo de pescado, es importante mencionar que en el informe de la consulta mixta de expertos FAO/OMS sobre los riesgos y los beneficios de su consumo, se concluyó que ante eventos de contaminación ambiental en pescado, restringir por completo el consumo no debería ser una acción de carácter permanente para disminuir el riesgo en la salud de una población. Esto gracias a beneficios como el aporte energético, de proteínas y de nutrientes, como los ácidos grasos poliinsaturados de cadena larga n-3 (AGPICLn3), que son característicos de este tipo 
de proteína animal; asimismo, porque en mujeres en edad fértil el consumo de pescado reduce el riesgo de deficiencias en el desarrollo neurológico en la progenie, siempre que la exposición materna por consumo de pescado a contaminantes ambientales, como las dioxinas o los metales pesados, no supere el valor de la ingesta mensual provisional tolerable. Adicionalmente, otros factores que pueden potenciar la ocurrencia de efectos adversos en la salud por el consumo de pescado con contenido de metales pesados son el estado nutricional, un deficiente acceso a servicios públicos básicos y un nivel de ingresos económicos bajos. Figueroa, Caicedo, Echeverry, Peña y Méndez (2017) demostraron que el mayor consumo de pescado en el oriente de la ciudad de Cali está asociado a un bajo nivel de ingresos económicos y a un bajo nivel de escolaridad. En ese sentido, la encuesta poblacional permitió identificar que existen condicionantes de tipo socioeconómicos, como bajos ingresos económicos (la media de ingresos de la población fue de 0,4 salarios mínimo mensuales legales vigentes), bajo nivel educativo (el $86 \%$ de las participantes solo contaba con estudios de básica primaria) y un acceso limitado a servicios de agua y saneamiento, que aumentan la vulnerabilidad social al riesgo potencial en salud. Estos condicionantes limitan la disponibilidad y la accesibilidad de las madres lactantes a una cantidad y calidad idónea de alimentos, por lo que encuentran en los peces del río Cauca una fuente de proteína de fácil acceso y de bajo costo, lo que favorece la exposición a los contaminantes evaluados. Por lo tanto, la intervención que se requiere en la zona debe enmarcarse en el ámbito de políticas integrales que tengan en cuenta los contextos de los territorios y disminuyan las inequidades ambientales y en salud de estas comunidades vulnerables.

\section{Conclusiones}

Este estudio evidencia la grave problemática ambiental asociada al deterioro de la cuenca del río Cauca, que exige la implementación de intervenciones a niveles que impacten la calidad del recurso y, en consecuencia, la salud de la población que se expone a este río.

La evaluación de riesgo muestra que en el corregimiento de El Hormiguero y en la Comuna 6 el riesgo no cancerígeno presenta valores intolerables, por lo que se deben implementar medidas urgentes de mitigación. Asimismo, los valores de la PTWI muestran que el consumo semanal de mercurio asociado a la ingesta de pescado supera los valores establecidos $\left(<5 \mu \mathrm{g} / \mathrm{kg}^{-1}\right.$ de peso corporal) por el Comité Mixto FAO/OMS de Expertos en Aditivos Alimentarios.

\section{Declaración de conflicto de intereses}

En relación a este manuscrito, los autores manifiestan que no existe ningún conflicto de interés relacionado con la financiación del estudio ni de ningún otro tipo. El estudio contó con el aval del Comité de Ética de la Universidad del Valle y el apoyo de los centros de salud del corregimiento de El Hormiguero y la Comuna 6, del oriente de Cali.

\section{Agradecimientos}

Los autores agradecen a los habitantes de cada una de las zonas evaluadas, por su disposición para la resolución de las encuestas poblacionales y la recolección de las muestras. 


\section{Referencias}

Al-Saleh, I., Shinwari, N., Mashhour, A., Mohamed, G., y Rabah, A. (2011). Heavy metals (lead, cadmium and mercury) in maternal, cord blood and placenta of healthy women. International Journal of Hygiene and Environmental Health, 214(2), 79-101. https://doi.org/10.1016/j.ijheh.2010.10.001

Alvarez, S., Jessick, A., Palacio, J., y Kolok, A. (2012). Methylmercury concentrations in six fish species from two Colombian rivers. Bulletin of Environmental Contamination and Toxicology, 88(1), 65-68. https://doi.org/10 .1007/s00128-011-0458-x

Alvarez, S., Kolok, A., Jimenez, L., Granados, C., y Palacio, J. (2012). Mercury concentrations in muscle and liver tissue of fish from marshes along the Magdalena river, Colombia. Bulletin of Environmental Contamination and Toxicology, 89(4), 836-840. https://doi.org/10.1007/s00128-012-0782-9

Burbure, C. de, Buchet, J., Leroyer, A., Nisse, C., Haguenoer, J., Mutti, A., y Bernard, A. (2006). Renal and neurologic effects of cadmium, lead, mercury, and arsenic in children: Evidence of early effects and multiple interactions at environmental exposure levels. Environmental Health Perspectives, 114(4), 584-590. https://doi.org/10.1289/ ehp.8202

Castro, M., y Méndez, M. (2008). Heavy metals: Implications associated to fish consumption. Environmental Toxicology and Pharmacology, 26(3), 263-271. https://doi.org/10.1016/j.etap.2008.06.001

Cordy, P., Veiga, M., Salih, I., Al-Saadi, S., Console, S., Garcia, O., y Roeser, M. (2011). Mercury contamination from artisanal gold mining in Antioquia, Colombia: The world's highest per capita mercury pollution. Science of the Total Environment, 410-411, 154-160. https://doi.org/10.1016/j.scitotenv.2011.09.006

Duque, G., Marrugo, J., Suárez, H., y Olivares, M. (2016). Evaluación de riesgo demercurio en peces de aguas continentales en Colombia. Bogotá: Instituto Nacional de Salud.

Echeverry, G., Zapata, A., Páez, M., Méndez, F., y Peña, M. (2015). Valoración del riesgo en salud en un grupo de población de Cali, Colombia, por exposición a plomo, cadmio, mercurio, ácido 2,4-diclorofenoxiacético y diuron, asociada al cosumo de agua potable y alimentos. Biomédica, 35, 110-19. https://doi.org/10.7705/biomedica.v $35 \mathrm{i} 0.2464$

El-Moselhy, K., Othman, A., El-Azem, H., y El-Metwally, M. (2014). Bioaccumulation of heavy metals in some tissues of fish in the Red Sea, Egypt. Egyptian Journal of Basic and Applied Sciences, 1(2), 97-105. https://doi.org/10 .1016/j.ejbas.2014.06.001

European Food Safety Authority. (2004). Opinion of the Scientific Panel on Contaminants in the Food Chain on a request from the Commission related to mercury and methylmercury in food. Oslo: Autor.

Figueroa, R., Caicedo, D., Echeverry, G., Peña, M., y Méndez, F. (2017). Condición socioeconómica, patrones de alimentación y exposición a metales pesados en mujeres en edad fértil de Cali, Colombia. Biomédica, 37(3), 341352. https://doi.org/10.7705/biomedica.v37i3.3286

García, E., Pérez, B., Fernández, M., Pérez, M., Gil, E., de Paz, C., ... Aragonéz, N. (2011). Mercury, lead and cadmium in human milk in relation to diet, lifestyle habits and sociodemographic variables in Madrid (Spain). Chemosphere, 85(2), 268-276. https://doi.org/10.1016/j.chemosphere.2011.05.029

Gil, M., Torres, A., Harvey, M., y Esteves, J. (2006). Heavy metals in marine organisms from the coastal zone of continental Argentine Patagonia. Revista de Biologia Marina y Oceanografia, 41(2), 167-176. https://doi.org/ $10.4067 /$ S0718-19572006000200004

Kwok, K., Liang, Y., Wang, H., Dong, H., Leung, Y., y Wong, H. (2014). Bioaccumulation of heavy metals in fish and ardeid at Pearl River Estuary, China. Ecotoxicology and Environmental Safety, 106, 62-67. https://doi.org/10.1 016/j.ecoenv.2014.04.016

Mancera, J., y Álvarez, R. (2006). Current state of knowledge of the concentration of mercury and other heavy metals in fresh water fish in Colombia. Acta Biológica Colombiana, 11(1), 3-23. https://doi.org/doa.org/toc/1900-1 $649 / 11 / 0$

Márquez, H., Torres, A., Macías, E., Hernández, A., Martínez, I., Morales, J., y Sánchez, I. (2016). Estimación de riesgo de exposición a metales pesados por consumo de plecos (Pterygoplichthys spp.) en infantes de comunidades 
ribereñas de los ríos Grijalva y Usumacinta, México. Revista Internacional de Contaminacion Ambiental, 32(2), 153-164. https://doi.org/10.20937/RICA.2016.32.02.02

Marrugo, J., Benitez, N., y Olivero, J. (2008). Distribution of mercury in several environmental compartments in an aquatic ecosystem impacted by gold mining in northern Colombia. Archives of Environmental Contamination and Toxicology, 55(2), 305-316. https://doi.org/10.1007/s00244-007-9129-7

Marrugo, J., Verbel, J., Ceballos, L., y Benitez, N. (2008). Total mercury and methylmercury concentrations in fish from the Mojana region of Colombia. Environmental Geochemistry and Health, 30(1), 21-30. https://doi.org/10.1 007/s10653-007-9104-2

Mejías, J., y Jerez, J. (2006). Guia para la toma de muestras de residuos de plaguicidas: Agua, sedimento y suelo. Temuco: Inia.

Mendoza, C., González, L., y Benítez, N. (2007). Concentracio\#n de mercurio y zinc en los suelos superficiales aledan\# os al basurero de Navarro de la ciudad de Cali. Determinacio\#n de su distribucio\#n espacial. Revista de La Facultad de Ciencias-Universidad del Valle, 11, 26-31.

Mercosur. (2014). Reglamento te\#cnico Mercosur sobre li\#mites ma\#ximos de contaminantes inorga\#nicos en alimentos. Asunción: Autor.

Monroy, M., Maceda, A., y Sostoa, A. de. (2014). Metal concentration in water, sediment and four fish species from Lake Titicaca reveals a large-scale environmental concern. Science of the Total Environment, 487(1), 233-244. h ttps://doi.org/10.1016/j.scitotenv.2014.03.134

Office of Research and Development, National Center for Environmental Assessment. (1987). Toxicological review of cadmium. Washington, D. C.: Autor.

Office of Research and Development, National Center for Environmental Assessment. (1989). Risk Assessment Guidance for Superfund. Volume I: Human Health Evaluation Manual (Part A) (Vol. I). Washington, D. C.: Autor.

Office of Research and Development, National Center for Environmental Assessment. (1996). Microwave Assisted Acid Digestion of Siliceous and Organically Based Matrices. Washington, D. C.: Autor.

Office of Research and Development, National Center for Environmental Assessment. (1998). Method 7010: Graphite Furnace Atomic Absorption Spectrophotometry. Washington, D. C.: Autor.

Office of Research and Development, National Center for Environmental Assessment. (2001). Toxicological Review of Mercury and Methylmercury. Washington, D. C.: Autor.

Office of Research and Development, National Center for Environmental Assessment. (2014). Toxicological Review of Lead. Washington, D. C.: Autor.

Organización de las Naciones Unidas para la Agricultura y la Alimentación (FAO). (2013). Codex General Standard for Contaminants and Toxins in Food and Feed. Roma: Autor.

Páez, M., Uribe, M., Díaz, S., Castro, R., Barbosa, E., Carvajal, N., y Londoño, A. (2011). Evaluación de riesgo en humanos por plaguicidas en tomate cultivado con sistemas tradicional y BPA (Buenas Prácticas Agrícolas). Revista Facultad de Ciencias Naturales y Exactas Universidad Del Valle, 15, 153-167.

Pollack, A., Sjaarda, L., Ahrens, K., Mumford, S., Browne, R., Wactawski, J., y Schisterman, E. (2014). Association of cadmium, lead and mercury with paraoxonase 1 activity in women. PLOS ONE, 9(3). https://doi.org/10.137 1/journal.pone.0092152

Programa Ambiental del Caribe (PNUMA). (2008). Guia para el muestreo, preparación y análisis de contaminantes orgánicos en muestras ambientales (agua, suelos/sedimentos y biota). Kingston: Autor.

Raissy, M., y Ansari, M. (2014). Health risk assessment of mercury and arsenic associated with consumption of fish from the Persian Gulf. Environmental Monitoring and Assessment, 186(2), 1235-1240. https://doi.org/10.100 7/s10661-013-3452-4

Storelli, M. (2008). Potential human health risks from metals ( $\mathrm{Hg}, \mathrm{Cd}$, and $\mathrm{Pb}$ ) and polychlorinated biphenyls (PCBs) via seafood consumption: Estimation of target hazard quotients (THQs) and toxic equivalents (TEQs). Food and Chemical Toxicology, 46(8), 2782-2788. https://doi.org/10.1016/j.fct.2008.05.011 
Suárez, L., y Aristizabal J. (2013). Mercury and gold mining in Colombia: A failed state. Universitas Scientiarum, 18(1), 33-49.

Vieira, C., Morais, S., Ramos, S., Delerue, D., y Oliveira, M. (2011). Mercury, cadmium, lead and arsenic levels in three pelagic fish species from the Atlantic ocean: Intra and inter specific variability and human health risks for consumption. Food and Chemical Toxicology, 49(4), 923-932. https://doi.org/10.1016/j.fct.2010.12.016

Vivas, A., Arboleda, M., Sanchez, R., Benitez, N., Bravo, E., Soto, A., y Larmat, F. (2014). Mutagenicity evaluation caused by heavy metals found in Cauca river water in the city of Cali, Colombia. Revista Colombiana de Quimica, 43(Mi), 18-24. https://doi.org/10.15446/rev.colomb.quim.v43n2.53119

\section{Notas}

* Artículo de investigación Este artículo es producto del proyecto Estrategias para la Recuperación y Manejo Integrado del Recurso Hídrico en las Cuencas del Cauca y Dagua, en el Valle del Cauca, financiado con recursos del Sistema General de Regalías del Valle del Cauca (SGR).

\section{Licencia Creative Commons CC BY 4.0}

Cómo citar este artículo: Zapata Rivera, A. M., Méndez, F., Páez, M. I., Abrahams Chow, N., Ordoñez, J., y Artunduaga, Y. P. (2018). Estudio exploratorio de evaluación de riesgo en la salud de madres lactantes por consumo de pescado contaminado del río Cauca, en el Valle del Cauca (Colombia). Ambiente y Desarrollo, 22(43). https://doi.org/10.11144/Javeriana.ayd22-43.eeer 